\section{EFFECT OF COOLING-AGITATION ON POLYMORPHIC} TRANSFORMATION OF L-GLUTAMIC ACID

C. Cashell ${ }^{1,2}$ B.K. Hodnett ${ }^{1,2}$ D. Corcoran ${ }^{1,3}$

University of Limerick MSSI Castletroy LIMERICK NONE IRELAND

${ }^{1}$ Materials and Surface Science Institute, University of Limerick ${ }^{2}$ Chemical and Environmental Sciences Department, University of Limerick ${ }^{3}$ Physics Department, University of Limerick

L-glutamic acid (L-Glu) can crystallize in two polymorphic structures, depending on the crystallization conditions employed. The metastable granular $\alpha$-form crystallizes first, and subsequently undergoes solution-mediated transformation to the stable plate-like $\beta$-form. Previous work shows that tailor-made additives such as ionic surfactants, 1,5-dicarboxylic acids and di-peptides kinetically stabilize the $\alpha$-form of L-Glu. It has been found that the low concentrations of L- $\alpha$-amino acids cause a significant reduction in the growth rate of the $\beta$-form. In this work, the effect of cooling-agitation on the polymorphic behavior of LGlu, in the absence of tailor-made additives was studied. The $\alpha$-form of L-Glu was synthesized by acidification of the monosodium salt. Supersaturated aqueous solutions of the $\alpha$-form at $80^{\circ} \mathrm{C}$ were recrystallized by cooling to $45^{\circ} \mathrm{C}$ with and without agitation. The effect of cooling-agitation speed on polymorphism of L-Glu was also investigated, and characterization of L-Glu crystallized at $45^{\circ} \mathrm{C}$ was performed using X-ray powder diffraction, Scanning Electron Microscopy, Differential Scanning Calorimetry and Raman Spectroscopy. Agitation was found to delay the $\alpha$ to $\beta$ transformation rate considerably, maintaining the $\beta$-L-Glu concentration at approximately $10 \%$. This stabilization of the $\alpha$ form increased significantly with agitation speed, and was most effective when pulsed agitation was employed. Alpha form stabilization of this magnitude has not been previously reported in the absence of additives, an observation that may be due to the absence of certain crystallographic faces of the $\alpha$-form, which facilitate nucleation of the $\beta$-form.

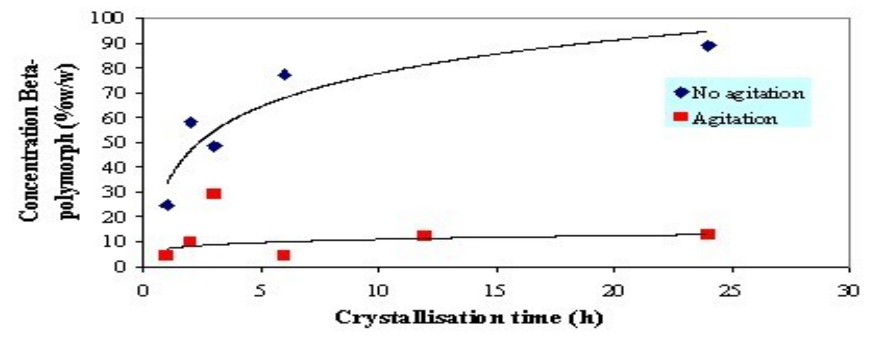

Keywords: POLYMORPHISM, GLUTAMIC ACID, AGITATION

\section{Acta Cryst. (2002). A58 (Supplement), C138}

POLYMORPHISM AND A PHASE TRANSITION IN $\mathrm{K}_{3} \mathrm{YB}\left(\mathrm{PO}_{4}\right)_{2}$

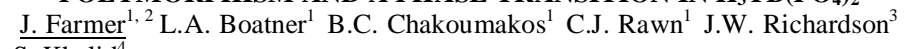
S. Khalid ${ }^{4}$

${ }^{1}$ Oak Ridge National Laboratory P.O. Box 2008, MS-6119 OAK RIDGE TN 37831 USA ${ }^{2}$ Baylor University, Chemistry Department, Waco, TX 76798, USA ${ }^{3}$ Argonne National Laboratory (ANL), Argonne, Il 60439, USA ${ }^{4}$ Brookhaven National Laboratory (BNL), Upton, NY 11973, USA

Alkali lanthanide double phosphates have been studied for use as longwavelength scintillators for $\gamma$-ray detection using $\mathrm{Si}$ photodiodes. These compounds exhibit layered crystal structures. Details of the crystal symmetry depend on the relative sizes of the lanthanide and alkali metals. Single-crystal $\mathrm{X}$-ray diffraction (XRD) and powder neutron diffraction (PND) have been used to study the structure at room temperature. $\mathrm{K}_{3} \mathrm{Yb}\left(\mathrm{PO}_{4}\right)_{2}$ crystallizes with a monoclinic unit cell, space group $P 2_{1} / \mathrm{m}$. The $\mathrm{Yb}$ ion is six-coordinated to the oxygen atoms of the phosphate groups with uint cell parameters $\mathrm{a}=7.372(1), \mathrm{b}$ $=5.589(1), \mathrm{c}=9.292(2) \AA$ and $\beta=91.03^{\circ}$. The $\mathrm{Yb}$ ion is seven coordinated with a slightly distorted capped trigonal prism (CTP) geometry. A high temperature phase was characterized using powder neutron diffraction and synchotron powder analysis. The phase transition occurs at $120^{\circ} \mathrm{C}$ with a transformation to the hexagonal $P-3$ space group symettry with a coordination reduction to six that is confirmed using EXAFS. This new structure is isostructural with the room-temperature form of $\mathrm{K}_{3} \mathrm{Lu}\left(\mathrm{PO}_{4}\right)_{2}$. High temperature PND and high temperature powder XRD have been used to study the thermal expansion of $\mathrm{K}_{3} \mathrm{Yb}\left(\mathrm{PO}_{4}\right)_{2}$ and indicate a large thermal expansion anisotropy. Research sponsored by the Division of Materials Sciences and by the Energy Efficiency and Renewable Energy Program, Office of Transportation Technologies, as part of the ORNL High Temperature Materials Laboratory User Program.

Keywords: HTXRD, PHASE TRANSITION, DOUBLE PHOSPHATE
Acta Cryst. (2002). A58 (Supplement), C138

\section{D STRUCTURES OF ACETYLCHOLINESTERASE COMPLEXED WITH POTENTIAL DRUGS FOR THE TREATMENT OF} ALZHEIMER'S DISEASE

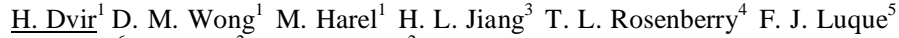
P. Camps ${ }^{6}$ I. Silman ${ }^{2}$ J. L. Sussman ${ }^{2}$

${ }^{1}$ Weizmann Institute of Science Department of Structural Biology Hertzel 100 REHOVOT 76100 ISRAEL ${ }^{2}$ Department of Neurobiology, Weizmann Institute of Science, Rehovot, ISRAEL ${ }^{3}$ State Key Laboratory of Drug Research, Shanghai Institute of Meteria Medica3, Chinese Academy of Sciences, Shanghai, China ${ }^{4}$ Department of Pharmacology, Mayo Clinic Jacksonville, Florida, USA ${ }^{5}$ Departament de Fisicoqumica, Facultat de Farmcia, Universitat de Barcelona, Barcelona, Spain ${ }^{6}$ Laboratori de Qumica Farmacutica, Facultat de Farmcia, Universitat de Barcelona, Barcelona, Spain

The enzyme acetylcholinesterase (AChE) functions in cholinergic synapses of central and peripheral nervous systems, where its principal role is termination of signal transmission by rapid hydrolysis of the neurotransmitter, acetylcholine (ACh). Inhibition of AChE is the only approved treatment for the symptoms of Alzheirmer's disease. We have studied the interactions of AChE with certain potential drugs based on the X-ray structures of their complexes with torpedo californica $\mathrm{AChE}$ and their experimental affinities measured in solution. We show that a family of natural alkaloids, called huperzines, leads to a disruption of the active site of AChE, by causing a conformational change in part of the catalytic machinery. Huprine $\mathrm{X}$ is a novel AChE inhibitor that was derived by combining active parts from two different know AChE inhibitors, huperzine and tacrine. It was shown that it binds AChE significantly tighter than both of them. The crystal structure of the complex shows that it behaves as a hybrid within the active site of $\mathrm{AChE}$, maintaining features from its two 'ancestors'.

Keywords: ACETYLCHOLINESETERASE, ALZHEIMER, HUPERZINE

\section{Acta Cryst. (2002). A58 (Supplement), C138}

\section{POLARITY FORMATION BY IN-DIFFUSION OF DYE MOLECULES} INTO CHANNELS OF AN ORGANIC ZEOLITE

$\underline{\text { T. Hertzsch }}^{1,2}$ S. Kluge ${ }^{1}$ E. Weber ${ }^{2}$ F. Budde ${ }^{1}$ J. Hulliger

${ }^{1}$ University of Berne Department of Chemistry And Biochemistry Freiestrasse 3 BERNE 3012 SWITZERLAND ${ }^{2}$ TU Bergakademie Freiberg; Institute of Organic Chemistry, Leipziger Strasse 29, D-09599 Freiberg, Germany

Channel forming organic zeolite materials are of great interest, because such host structures may provide van der Waals-type inner surfaces as compared to ionic surfaces in inorganic zeolites. Inclusion compounds of tris(ophenylenedioxy)cyclotriphospazene (TPP) allow to prepare empty but thermally fairly stable $\left(\mathrm{T}<150^{\circ} \mathrm{C}\right)$ channels $(5 \AA)$, which show effects of sorption/desorption when exposed to guest molecules/atoms such as tetrahydrofuran (THF), benzene and xenon. Because channels of TPP are built by a staggered trigonal coordination of phenylene rings, guest molecules showing $\pi$-acceptor properties can give rise to an exchange of template by donor/acceptor di-substituted $\pi$-conjugated frameworks. When organic molecules such as 4-N,N-dimethlyamino-4-nitrostilbene or other dipolar compounds were kept in sealed ampoules in the presence of single crystals of TPP-THF for a few days at $\mathrm{T} 120^{\circ} \mathrm{C}$, an exchange of THF vs new guest molecules was observed ${ }^{1}$. Dye molecules were entering needle shaped TPPTHF crystals from both capping faces, filling zeolite crystals up to the center. Because TPP-THF crystals did not show empty channels prior to in-diffusion, we conclude that a mechanism of counter-diffusion is active in TPP-THF crystals. Stained, TPP-zeolite materials showed a SHG effect indicative for dye molecules recognising the surface of the zeolite. These findings can be understood because of a general principle of polarity formation, saying that vector properties are preserved at a crystal-nutrient interface: In-coming dipolar molecules exceed an orientational selection with respect of entering preferably the acceptor or donor group first.

References

[1] Hertzsch T., Kluge S., Weber E., Hulliger J., Adv. Mater. (2001), 13: 64.

Keywords: ORGANIC ZEOLITE, INCLUSION COMPOUNDS, MOLECULAR RECOGNITION 